\title{
Heuristics for the Economic Production Quantity Problem under Restrictions on Production and Maintenance Time
}

\author{
Qi Xu${ }^{1}$ and Jianteng $X u^{2}$ \\ ${ }^{1}$ School of Medical Information Engineering, Jining Medical University, Rizhao 276826, China
}

${ }^{2}$ School of Management, Qufu Normal University, Rizhao 276826, China

Correspondence should be addressed to Jianteng Xu; jiantengxu@163.com

Received 12 May 2016; Accepted 25 August 2016

Academic Editor: Inmaculada T. Castro

Copyright (c) 2016 Q. Xu and J. Xu. This is an open access article distributed under the Creative Commons Attribution License, which permits unrestricted use, distribution, and reproduction in any medium, provided the original work is properly cited.

This paper proposes an economic production quantity problem with the maximal production run time and minimal preventive maintenance time over a finite planning horizon. The objective is to find the efficient production and maintenance policy to minimize the total cost composed of production, maintenance, shortages, and holding costs under the restriction on the production run time and the preventive maintenance time. The production and maintenance decisions include the production and maintenance frequencies and the production run and the maintenance time. The variability and the boundedness of the production run and maintenance time make the problem difficult to solve. Two heuristic algorithms are developed using different techniques based on the optimal properties of the relaxed problem. The performance comparison between the two algorithms is illustrated by numerical examples. The numerical results show that, for the most part, there exists a heuristic algorithm which is more effective than the other.

\section{Introduction}

The economic production quantity (EPQ) is a fundamental inventory control problem, and it is still widely used by many industries today [1]. Some literature concentrates on the classical EPQ problem and its extensions, such as Chung and Huang [2], Cárdenas-Barrón [3], Jeang [4], Lee and Yang [5], Mezei and Björk [6], and Lee et al. [7]. In these extensions of the classical EPQ problem, one of the assumptions is that the production process is not interrupted. However, in reality, the production process is usually interrupted by some outside and inside reasons. For example, in some cities of China, such as Shanghai and Guangzhou, the restriction policy on electricity power supply is usually imposed on the manufacturing industries when the demand of the electricity power exceeds the supply. On the other hand, in some manufacturing industries, it is necessary to stop production and give the preventive maintenance (PM) or perform the corrective maintenance $(\mathrm{CM})$ when the production facility breaks down. The production interruption brings new problems to the production decisions of the manufacturing industries and has received more and more attention.
The effects of machine breakdown and CM (or rework) on the production decision were studied by Groenevelt et al. [8], Abboud [9], Lin and Gong [10], Chiu et al. [11], Chiu et al. [12], Prakash et al. [13], and Ting and Chung [14], among others. Compared with CM, PM can effectively avoid the machine breakdown. Meller and Kim [15] studied the impact of PM on system cost and optimal inventory level. Giri and Dohi [16] considered a flexible unreliable manufacturing system with random machine failure and repair time and formulated a generalized EPQ model to determine the optimal production rate and production quantity. Liao et al. [17], and Liao and Sheu [18] integrated PM into the EPQ model and considered the imperfect repair of the preventive maintenance with a random failure rate. Lee [19] studied the production and maintenance strategy for a deteriorating production system in which the products were sold with free minimal repair warranty. He developed a framework which could simultaneously determine the optimal production run length and maintenance schedule. Lin et al. [20] investigated an imperfect production system with inspection errors, preventive maintenance errors, and minimal repairs. Sana [21] considered an imperfect production system with 


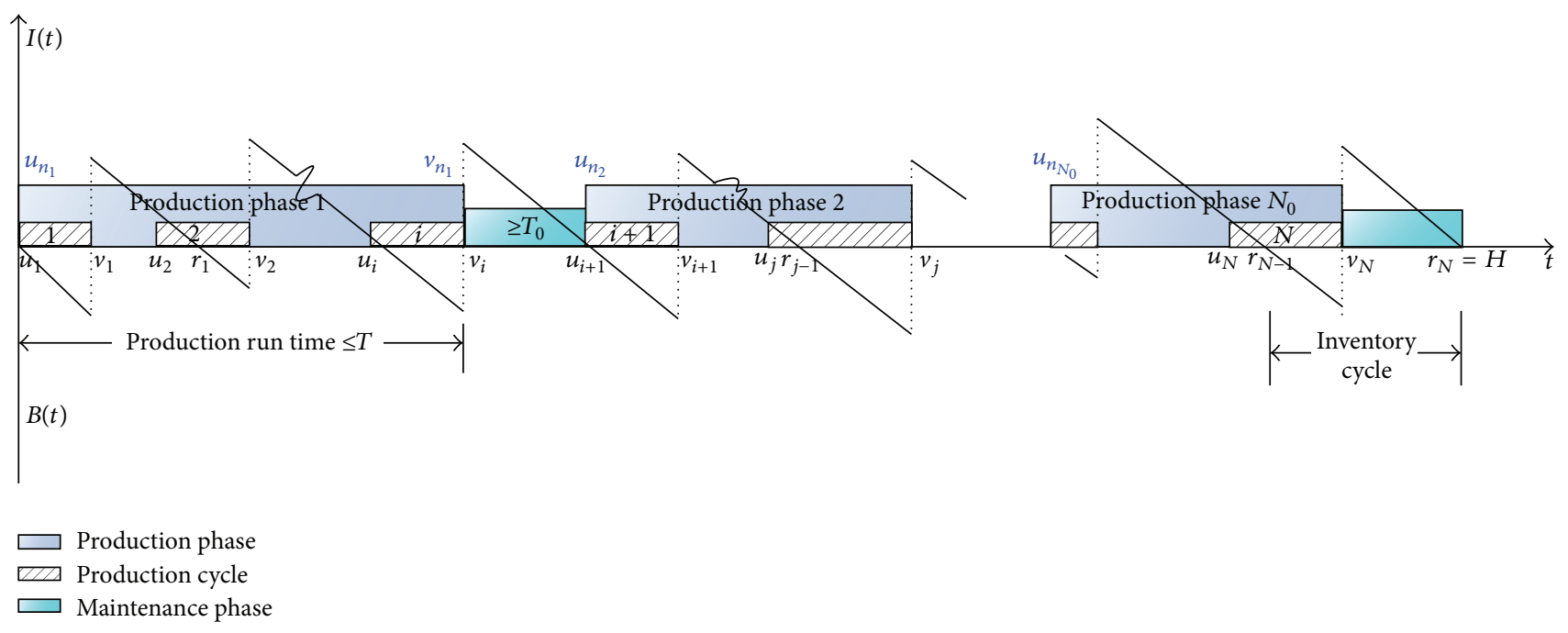

FIGURE 1: Graphical description for production and inventory process.

PM for products sold with free minimal repair warranty and proposed the optimal buffer level and production run time. Widyadana and Wee [22], Wee and Widyadana [23], and Wee and Widyadana [24] developed EPQ models for deteriorating items with stochastic preventive maintenance time. Liao [25] considered a deteriorating production system with imperfect and perfect maintenance and developed an EPQ model to determine the optimal run time. Tsao [26] proposed a piecewise nonlinear optimization algorithm to solve the optimal replenishment frequencies for the productioninventory problem with maintenance, variable setup costs, and trade credits. Gan et al. [27] focused on the effects of maintenance, buffer inventory, and spare parts inventory on the minimization of the long-term expected cost rate for a production system. Emami-Mehrgani et al. [28] studied the impact of human error on repairable manufacturing systems over an infinite planning horizon. Nourelfath et al. [29] developed an optimization model for a joint production plan, the maintenance policy, and quality process in a multiperiod multiproduct capacitated lot-sizing context.

Different from the above literature, this paper proposes a generalized EPQ model under the restrictions on production run time and maintenance time over a finite planning horizon. The restrictions on production and maintenance time are embodied by the assumptions that the production run time can not exceed a maximum value once the production facility is turned on, and the maintenance time is not less than a minimum value. The production and maintenance decisions include both the production and maintenance frequencies and the production run and the maintenance time. By relaxing the constructed EPQ model and analyzing some optimal properties, this paper designs two heuristic algorithms using different techniques and compares their performance by numerical examples.

The rest of the paper is organized as follows. Section 2 describes the problem and develops a mixed integer quadratic programming model. Section 3 presents some theoretical results of the relaxation model which help to design the heuristic algorithms. Section 4 designs two heuristic algorithms to solve this problem using different techniques. Section 5 compares these two heuristic algorithms by numerical experiments. Finally, conclusions are proposed in Section 6.

\section{Problem Description and Mathematical Model}

2.1. Problem Description. The problem considered in this paper can be described as follows: the manufacturer needs to meet the demand of customers over a finite planning horizon in a batch production system with one production facility. In order to insure the facility against breakdown, the manufacturer performs PM to the facility. The restrictions on production and maintenance are expressed by the maximal production run time and the minimal preventive maintenance time. In other words, the production run time of each production phase can not exceed a maximal value. And the maintenance time of each preventive maintenance phase can not be less than a minimal value. The inventory levels at the beginning and the end of the planning horizon are zero. Shortages are allowed and all shortages are backlogged. The objective of this problem is to determine an effective production and maintenance policy including the production and maintenance frequencies and the production run time in order to minimize the total relevant cost composed of production, maintenance, shortages, and holding costs over the finite planning horizon.

In order to make the problem much clearer, we define some notions including production phase, production run time, maintenance phase, production cycle, and inventory cycle, which are described in Figure 1. It can be seen that the constraints on production divide the planning horizon into several production phases and maintenance phases. The production phase starts at the time when the production facility is turned on and ends at the time when the production facility is turned off. The length of each production phase is 
called a production run time. The maintenance phase starts at the time when the production facility is turned off and ends at the time when the production facility is turned on again. In each production phase, there are several production cycles and inventory cycles. A production cycle starts at the time when a batch is produced and ends at the time when the batch is finished. An inventory cycle is the interval between the times when the inventory level drops to zero successively. The shortage occurs when the inventory level first drops to zero until a batch is finished. Once a batch is finished, the products are transported to the warehouse, and the inventory level reaches the maximum.

2.2. Mathematical Model. The notations in Notations section are used to propose the mathematical model.

Based on the notations, we can conclude that the interval of $i$ th production cycle is $\left[u_{i}, v_{i}\right]$ and the interval of $i$ th inventory cycle is $\left[r_{i-1}, r_{i}\right], i=1, \ldots, N$. The behavior of inventory system is depicted in Figure 1 . The interval of $k$ th production phase is $\left[u_{n_{k}}, v_{n_{k}}\right]$, where $n_{k} \in[1, N], k=$ $1, \ldots, N_{0}$. In addition, letting $B(t)$ be the shortages quantity at time $t$, we easily have $B(t)=d\left(t-r_{i-1}\right), t \in\left[r_{i-1}, v_{i}\right], i=$ $1, \ldots, N$. Letting $I(t)$ be the on-hand inventory level at time $t$, we have $I(t)=d\left(r_{i}-t\right), t \in\left[v_{i}, r_{i}\right], i=1, \ldots, N$. Therefore, the total shortages during $i$ th inventory cycle (denoted by $B_{i}$ ) is $B_{i}=\int_{r_{i-1}}^{v_{i}} B(t) d t=d\left(v_{i}-r_{i-1}\right)^{2} / 2$, and the total on-hand inventory from $v_{i}$ to $r_{i}$ during $i$ th inventory cycle (denoted by $\left.I_{i}\right)$ is $I_{i}=d\left(r_{i}-v_{i}\right)^{2} / 2, i=1, \ldots, N$.

Consequently, the problem considered in this paper (denoted by $P$ ) can be formulated as

$$
\begin{array}{ll}
P: \min & \mathrm{TC}\left(N_{0}, N, \mathbf{u}, \mathbf{v}, \mathbf{r}\right) \\
& =N_{0} S_{0}+N S+c d H+\sum_{i=1}^{N}\left(b B_{i}+h I_{i}\right) \\
\text { s.t. } \quad & p\left(v_{i}-u_{i}\right)=B\left(v_{i}\right)+I\left(v_{i}\right), \quad i=1, \ldots, N, \\
& v_{n_{k}}-u_{n_{k}} \leq T, \\
& \quad k=1, \ldots, N_{0}-1, n_{k} \in[1, N], \\
& u_{n_{k+1}}-v_{n_{k}} \geq T_{0}, \quad k=1, \ldots, N_{0}, n_{k} \in[1, N], \\
& u_{i} \geq v_{i-1}, \quad i=2, \ldots, N, \\
& r_{i-1} \leq v_{i} \leq r_{i}, \quad i=1, \ldots, N .
\end{array}
$$

Note that the total production quantity is equal to the total demand over the planning horizon, so the variable production cost $c d H$ is a constant in the objective function of model $P$. Constraint (2) represents that the production quantity of $i$ th production cycle is equal to the demand of interval $\left[r_{i-1}, r_{i}\right]$. Constraint (3) represents that the production run time in $k$ th production phase can not exceed maximum value $T$. It can be seen that $u_{n_{k}}$ must be one of the start times $u_{i}$ among the production cycles; hence $n_{k} \in[1, N]$ with $n_{1}=1$, and $v_{n_{k}}=v_{n_{k+1}-1}$. Constraint (4) represents that $k$ th preventive maintenance time is not less than $T_{0}$. Constraints (5)-(6) describe the relations among the decision variables.

It can be seen that model $P$ is a complex mixed integer quadratic programming problem where decision variables $N_{0}$ and $N$ are integers and others are continuous real values. From the aspect of the integer variables, the smallest values of $N_{0}$ and $N$ are best. However, the total cost of the production phases with the smallest $N_{0}$ and $N$ may not be the minimal one. The balance of these two aspects and the variable production run time of each production phase make the optimal solution of the model $P$ difficult to find. In order to derive an effective solution procedure, we first investigate the properties of the relaxation model in the next section.

\section{Theoretical Results of the Relaxation Model}

In this section, we investigate the relaxation problem of model $P$ and analyze its optimal properties which will help us design two heuristic algorithms in the following section. Ignoring constraints (3)-(4) in model $P$, we formulate the relaxation model (denoted by $R P$ ) as

$$
\begin{aligned}
R P: \min & \operatorname{TC}^{\prime}(N, \mathbf{v}, \mathbf{r})=N S+c d H+\sum_{i=1}^{N}\left(b B_{i}+h I_{i}\right) \\
\text { s.t. } & p\left(v_{i}-v_{i-1}\right)=B\left(v_{i}\right)+I\left(v_{i}\right), \\
& \quad i=1, \ldots, N, \\
& r_{i-1} \leq v_{i} \leq r_{i}, \quad i=1, \ldots, N .
\end{aligned}
$$

It can be seen that there is only one production phase in model $R P$. Hence, we easily conclude that $u_{i}=v_{i-1}$ with $v_{0}=$ $0, i=1, \ldots, N$ in model $R P$. It means that model $R P$ satisfies constraint (5). Next, we propose the optimal properties of model $R P$.

For given $N$, we first ignore constraint (9) and reduce model $R P$ to an equality-constrained problem whose Lagrange function is

$$
\begin{aligned}
L(\mathbf{v}, \mathbf{r}, \boldsymbol{\omega})= & N S+c d H+\sum_{i=1}^{N}\left(b B_{i}+h I_{i}\right) \\
& -\sum_{i=1}^{N} \omega_{i}\left[p\left(v_{i}-v_{i-1}\right)-B\left(v_{i}\right)-I\left(v_{i}\right)\right],
\end{aligned}
$$

where $\omega_{i}, i=1,2, \ldots, N$, is the Lagrangian multiplier of constraint (8).

By taking the first partial derivatives of $L(\mathbf{v}, \mathbf{r}, \boldsymbol{\omega})$ with respect to $v_{i}, r_{i}$, and $\omega_{i}$ and setting the results to zero, we obtain

$$
\begin{aligned}
& \frac{\partial L(\mathbf{v}, \mathbf{r}, \boldsymbol{\omega})}{\partial v_{i}}=0, \quad i=1, \ldots, N, \\
& \frac{\partial L(\mathbf{v}, \mathbf{r}, \boldsymbol{\omega})}{\partial r_{i}}=0, \quad i=1, \ldots, N, \\
& p\left(v_{i}-v_{i-1}\right)=B\left(v_{i}\right)+I\left(v_{i}\right), \quad i=1, \ldots, N .
\end{aligned}
$$


Simplifying the above equations, we obtain

$$
\begin{array}{r}
h(p-d)\left(r_{i}-v_{i}\right)+b d\left(v_{i}-r_{i-1}\right)-b p\left(v_{i+1}-r_{i}\right)=0, \\
i=1, \ldots, N, \\
p\left(v_{i}-v_{i-1}\right)=d\left(r_{i}-r_{i-1}\right), \quad i=1, \ldots, N .
\end{array}
$$

Since $v_{0}=r_{0}=0$, if given $v_{1}$, we can calculate the value of variables $r_{i}$ and $v_{i+1}$ successively according to (13) and (12), $i=$ $1, \ldots, N$. Hence, we take variables $v_{i}$ and $r_{i}$ as the functions of $v_{1}$ and denote them as $v_{i}\left(v_{1}\right)$ and $r_{i}\left(v_{1}\right), i=1, \ldots, N$. Remembering that the inventory level is zero at the end of the finite planning horizon, we need to find the value of $v_{1}$ such that $r_{N}\left(v_{1}\right)=H$.

Theorem 1. There exists unique value of $v_{1}$ such that $r_{N}\left(v_{1}\right)=$ $H$ for model RP.

Proof. Let $v_{1}=0$; we can obtain $r_{1}=0$ from (13), $v_{2}=0$ from (12), and $r_{2}=0$ from (13). Following this alternate procedure, we can obtain $v_{i}(0)=r_{i}(0)=0, i=1, \ldots, N$. On the other hand, let $v_{1}=H$; we can obtain $r_{1}=H p / d>H$ and $v_{2}=$ $H[h(p-d)(p / d-1)+b d] /(b p)+r_{1}>H, \ldots, r_{N}>H$ from (12) and (13). That is, $r_{N}(0)<H$ and $r_{N}(H)>H$. So, there must exist some $v_{1}$ such that $r_{N}\left(v_{1}\right)=H$ in interval $[0, H]$. Let $F(x)=d\left(x-r_{i-1}\right)$; we have $F^{\prime}(x)=d>0$, which means that $F(x)$ is a strict increasing function of $x$. Note that $F\left(r_{i-1}\right)=$ 0 and $v_{i}-v_{i-1} \geq 0$; there exists unique $r_{i}\left(r_{i} \geq r_{i-1}\right)$ such that $F\left(r_{i}\right)=p\left(v_{i}-v_{i-1}\right)$. Similarly, let $G(x)=b p\left(x-r_{i}\right)$; we have $G^{\prime}(x)=b p>0$, which means that $G(x)$ is a strict increasing function of $x$. Since $G\left(r_{i}\right)=0$ and $h(p-d)\left(r_{i}-\right.$ $\left.v_{i}\right)+b d\left(v_{i}-r_{i-1}\right) \geq 0$, there exists unique $v_{i+1}\left(v_{i+1} \geq r_{i}\right)$ such that $G\left(v_{i+1}\right)=h(p-d)\left(r_{i}-v_{i}\right)+b d\left(v_{i}-r_{i-1}\right)$. Hence, there exists unique value of $v_{1}$ such that $r_{N}\left(v_{1}\right)=H$.

Now, we prove that the solution obtained from (12)-(13) satisfies constraint (9).

Theorem 2. The solution of (12)-(13) satisfies $r_{i-1} \leq v_{i} \leq$ $r_{i}, i=1, \ldots, N$.

Proof. We prove this theorem by mathematical induction. For $i=1, v_{1} \leq r_{1}$ can be obtained from (13) with $p \geq d$. From (12) with $h(p-d)\left(r_{1}-v_{1}\right)+b d\left(v_{1}-r_{0}\right)-b p\left(v_{2}-r_{1}\right)=0$, we can conclude that $v_{2} \geq r_{1}$. Hence, $r_{0} \leq v_{1} \leq r_{1}$ holds for $i=1$.

Assume that $r_{i-1} \leq v_{i} \leq r_{i}$ holds for $i=m$; that is, $r_{m-1} \leq$ $v_{m} \leq r_{m}$. We prove that it also holds for $i=m+1$. When $i=m$, we can conclude that $v_{m+1} \geq r_{m}$ from (12). For $i=m+1$, we have $p\left(v_{m+1}-v_{m}\right)=d\left(r_{m+1}-r_{m}\right)$ from (13). According to the assumption that $r_{m-1} \leq v_{m} \leq r_{m}$ and the fact that $v_{m+1}-v_{m} \leq$ $r_{m+1}-r_{m}$, we have $v_{m+1}-r_{m+1} \leq v_{m}-r_{m} \leq 0$. Consequently, $r_{m} \leq v_{m+1} \leq r_{m+1}$.

From the proof of Theorem 2, we can conclude the following corollary.

Corollary 3. The optimal solution of model RP satisfies $r_{i-1}$ $v_{i-1} \leq r_{i}-v_{i}, i=1, \ldots, N$.

Corollary 3 means that the storage time of $i$ th inventory cycle is an increasing function of $i, i=1, \ldots, N$.
Theorems 1 and 2 show that the optimal solution of model $R P$ can be uniquely determined.

Theorem 4. The optimal solution of model RP satisfies $r_{i}-$ $r_{i-1} \leq r_{i+1}-r_{i}, i=1, \ldots, N-1$; that is, the optimal inventory cycles of model RP are monotonically nondecreasing function of $i$.

Proof. From (12) and (13), we have

$$
\begin{aligned}
r_{i+1}- & r_{i}-\left(r_{i}-r_{i-1}\right)=\frac{p}{d}\left(v_{i+1}-v_{i}\right)-\left(r_{i}-r_{i-1}\right) \\
= & \frac{p}{d}\left[\frac{h(p-d)}{b p}\left(r_{i}-v_{i}\right)+\frac{b d}{b p}\left(v_{i}-r_{i-1}\right)+r_{i}-v_{i}\right] \\
& -\left(r_{i}-r_{i-1}\right) \\
= & \frac{h(p-d)}{b d}\left(r_{i}-v_{i}\right)+\left(1-\frac{p}{d}\right) v_{i}+\left(\frac{p}{d}-1\right) r_{i} \\
= & {\left[\frac{h(p-d)}{b d}+\frac{p}{d}-1\right]\left(r_{i}-v_{i}\right) \geq 0 . }
\end{aligned}
$$

Hence, we have $r_{i+1}-r_{i} \geq r_{i}-r_{i-1}$ for $i=1, \ldots, N-1$.

By applying the principle of optimality in Bellman [30], we have the following theorem.

Theorem 5. The objective function of model $R P, T C^{\prime}(N, \mathbf{v}, \mathbf{r})$, is a convex function of $N$.

The proof of Theorem 5 can be found in Appendix.

\section{Heuristic Algorithms}

On the basis of the optimal properties of model $R P$, we develop two heuristic algorithms using different techniques to determine the production and maintenance policy in this section.

Let $\left(N^{*}, \mathbf{v}^{*}, \mathbf{r}^{*}\right)$ be the optimal solution of model $R P$. We can obtain the approximate solution of model $P$ by following process:

(i) If $\left(N^{*}, \mathbf{v}^{*}, \mathbf{r}^{*}\right)$ satisfies the constraints on production run time and maintenance time, that is, constraints (3)-(4), then it is the feasible solution of model $P$.

(ii) Otherwise, we can obtain the approximate solution of model $P$ via the following approach:

(a) Find $i$ th production cycle in which end time $v_{i}^{*}$ is the first one to exceed $v_{0}+T$ in the optimal solution $\left(N^{*}, \mathbf{v}^{*}, \mathbf{r}^{*}\right)$ of model $R P$ and denote it by $i_{1}, i_{1}=1, \ldots, N^{*}$. Then, the first-production phase ends at $v_{i_{1}-1}^{*}$. The number of production cycles in the first-production phase is $i_{1}-1$. The first-maintenance phase starts at $v_{i_{1}-1}^{*}$ and ends at $v_{i_{1}-1}^{*}+T_{0}$.

(b) Set the start time of $i_{1}$ th production cycle $u_{i_{1}}$ to $v_{i_{1}-1}^{*}+T_{0}$. Recalculate the optimal solution of 
model $R P$ in $\left[r_{i_{1}-1}^{*}, H\right]$ with $v_{0}=v_{i_{1}-1}^{*}+T_{0}$ and $r_{0}=r_{i_{1}-1}^{*}$. Find $i$ th production cycle where $v_{i}^{*}$ is the first one to exceed $v_{0}+T$ and denote it by $i_{2}$. Then, the second-production phase ends at $v_{i_{2}-1}^{*}$. The number of production cycles in the second-production phase is $i_{2}-1$. The secondmaintenance phase starts at $v_{i_{2}-1}^{*}$ and ends at $v_{i_{2}-1}^{*}+T_{0}$.

(c) Keep on this process until the solution of each production phase satisfies the constraints on production run time and maintenance time. Then, the sets of the production phases and the optimal production cycles are the approximate solution of model $P$.

Let $S N_{0}$ be the index set of the first-production cycle in each production phase in the approximate solution of model $P$ obtained by the above approach and $\max \left\{S N_{0}\right\}$ be the maximal index in $S N_{0}$. We propose the above approach as an algorithm (denoted by $A_{1}$ ).

\section{Algorithm $A_{1}$}

Step 1. Input the values of $H, T, T_{0}, S_{0}, S, p, d, h, b$, and $c$. Set $r_{0}=0, v_{0}=0, N_{0}=1$, and $S N_{0}=\{1\}$.

Step 2. In interval $\left[r_{0}, H\right]$, calculate the optimal solution $\left(N^{*}, \mathbf{v}^{*}, \mathbf{r}^{*}\right)$ with $r_{N^{*}}=H$. Find $i$ th production cycle of $\left(N^{*}, \mathbf{v}^{*}, \mathbf{r}^{*}\right)$ in which end time $v_{i}^{*}$ is the first one to satisfy $v_{i}^{*}>v_{0}+T$, and let $m_{0}=i$. Go to Step 3 .

Step 3. If $m_{0}$ exists, record the first $\left(m_{0}-1\right)$ production and inventory cycles in $\left(N^{*}, \mathbf{v}^{*}, \mathbf{r}^{*}\right)$ as the solution of $N_{0}$ th production phase in model $P$. Then, let $N_{0}=N_{0}+1, S N_{0}=$ $S N_{0} \cup\left\{\max \left\{S N_{0}\right\}-1+m_{0}\right\}, r_{0}=r_{m_{0}-1}^{*}, v_{0}=v_{m_{0}-1}^{*}+T_{0}$, and go to Step 2. Otherwise, denote $\left(N^{*}, \mathbf{v}^{*}, \mathbf{r}^{*}\right)$ to be the solution of $N_{0}$ th production phase, stop.

The other approach to obtain the approximate solution of model $P$ is motivated by the following idea: if the length of finite planning horizon $H$ is not more than consecutive production run time $T$, then the optimal solution of model $R P$ is the optimal one of model $P$. Otherwise, divide the finite planning horizon into several production phases with the same length $T$ except for the last one. Denote the production phases by $\left[a_{j}, b_{j}\right]$; then we have $a_{j}=(j-1)\left(T+T_{0}\right), j=$ $1, \ldots,\left\lceil H /\left(T_{0}+T\right)\right\rceil$ and $b_{j}=a_{j}+T$ when $j=1, \ldots,\left\lceil H /\left(T_{0}+\right.\right.$ $T)\rceil-1$, and $b_{j}=H$ when $j=\left\lceil H /\left(T_{0}+T\right)\right\rceil$. Calculate the optimal solution of model $R P$ in $j$ th production phase with $v_{j, 0}=a_{j}, r_{j, 0}=r_{j-1, N_{j-1}}$, and $v_{j, N_{j}}=b_{j}$, where $r_{j-1, N_{j-1}}$ represents the end time of the last inventory cycle in $(j-1)$ th production phase. Hence, the set of each production phase optimal solution is the approximate solution of model $P$. This approach is described by heuristic Algorithm $A_{2}$.

\section{Algorithm $\mathrm{A}_{2}$}

Step 0. Input the values of $H, T, T_{0}, S_{0}, S, p, d, h, b$, and $c$.
Step 1. Compare $H$ with $T$. If $H \leq T$, then the optimal solution of model $P$ is the optimal solution of model $R P$; stop. Otherwise, set $j=1, r_{j, 0}=0$, and $v_{j, 0}=0$, and go to Step 2 .

Step 2. If $\left\lfloor H /\left(T_{0}+T\right)\right\rfloor=0$, then set $N_{0}=1$. Calculate the optimal solution of model $R P$ with $r_{j, N_{j}}=H$ in $[0, H]$, and denote it by $\left(N_{j}^{*}, \mathbf{v}_{j}^{*}, \mathbf{r}_{j}^{*}\right)$.

(i) If $v_{j, N_{j}^{*}}^{*} \leq T$, then the optimal solution $\left(N_{j}^{*}, \mathbf{v}_{j}^{*}, \mathbf{r}_{j}^{*}\right)$ is the optimal solution of model $P$. Stop.

(ii) If there exists some $i, 1 \leq i \leq N_{j}^{*}$ such that $v_{j, i}^{*}>T$, then resolve model $R P$ with $v_{j, N_{j}^{*}}^{*}=T$ in $[0, H]$. The total cost is the cost of new solution adding $S_{0}$ and the storage and shortage costs during $[T, H]$. Stop.

If $\left\lfloor H /\left(T_{0}+T\right)\right\rfloor>0$, then the following cases are considered:

(i) When $j<\left\lceil H /\left(T_{0}+T\right)\right\rceil$, go to Step 3 .

(ii) When $j=\left\lceil H /\left(T_{0}+T\right)\right\rceil$ and $v_{j, 0}+T<H$, then calculate the optimal solution and objective value $\mathrm{TC}_{j}\left(N_{j}^{*}, \mathbf{v}_{j}^{*}, \mathbf{r}_{j}^{*}\right)$ of model $R P$ with $v_{j, N_{j}^{*}}^{*}=v_{j, 0}+T$ in $\left[r_{j, 0}, H\right]$ and go to Step 5 .

(iii) When $j=\left\lceil H /\left(T_{0}+T\right)\right\rceil$ and $v_{j, 0}+T \geq H$, then calculate the optimal solution and objective value $\mathrm{TC}_{j}\left(N_{j}^{*}, \mathbf{v}_{j}^{*}, \mathbf{r}_{j}^{*}\right)$ of model $R P$ with $r_{j, N_{j}^{*}}^{*}=H$ in $\left[r_{j, 0}, H\right]$ and go to Step 5 .

Step 3. Calculate the optimal solution and objective value $\mathrm{TC}_{j}\left(N_{j}^{*}, \mathbf{v}_{j}^{*}, \mathbf{r}_{j}^{*}\right)$ of model $R P$ with $v_{j, N_{j}^{*}}^{*}=v_{j, 0}+T$ in $\left[r_{j, 0}, v_{j, 0}+\right.$ $T]$, and go to Step 4 .

Step 4. If $r_{j, N_{j}^{*}}^{*}=H$, go to Step 5 .

If $r_{j, N_{j}^{*}}^{*}<H$, set $j=j+1, r_{j, 0}=r_{j, N_{j}^{*}}^{*}$, and $v_{j, 0}=v_{j, N_{j}^{*}}^{*}+$ $T_{0}$, and go to Step 3 .

If $r_{j, N_{j}^{*}}^{*}>H$, calculate the optimal solution and objective value $\mathrm{TC}_{j}\left(N_{j}^{*}, \mathbf{v}_{j}^{*}, \mathbf{r}_{j}^{*}\right)$ of model $R P$ with $r_{j, N_{j}^{*}}^{*}=H$ in $\left[r_{j, 0}, H\right]$, and go to Step 5 .

Step 5. Set $N_{0}=j$. The solution obtained by Algorithm $A_{2}$ is the set of the optimal solutions in $N_{0}$ intervals. The total cost is the sum of cost $\mathrm{TC}_{j}\left(N_{j}^{*}, \mathbf{v}_{j}^{*}, \mathbf{r}_{j}^{*}\right), j=1, \ldots, N_{0}$, plus $N_{0} S_{0}$.

The performance of these two heuristic algorithms is investigated in the next section.

\section{Numerical Example}

In this section, a numerical example is provided to illustrate the applicability and performance of the heuristic Algorithms $A_{1}$ and $A_{2}$. The corresponding parameters are as follows: $H=$ $7, T=4, T_{0}=1, S_{0}=110, S=55, p=60, d=26, h=8$, $b=10$, and $c=3$.

Applying Algorithms $A_{1}$ and $A_{2}$, the optimal number of production cycles is 3 and 3 , and the corresponding total cost is 2744 and 2744 , respectively. It means that there is no 
TABLE 1: Comparison of running results of two heuristics.

\begin{tabular}{|c|c|c|c|c|c|c|c|}
\hline \multirow{2}{*}{ Parameters } & & & \multicolumn{5}{|c|}{$T_{0}$} \\
\hline & & & 0.1 & 0.5 & 1 & 1.5 & 2 \\
\hline \multirow{10}{*}{$H$} & \multirow{2}{*}{3} & $N$ & $(2,2)$ & $(2,2)$ & $(2,2)$ & $(2,2)$ & $(2,2)$ \\
\hline & & $T C$ & $(1134,1134)$ & $(1134,1134)$ & $(1134,1134)$ & $(1134,1134)$ & $(1134,1134)$ \\
\hline & \multirow{2}{*}{5} & $N$ & $(2,2)$ & $(2,2)$ & $(2,2)$ & $(2,2)$ & $(2,2)$ \\
\hline & & $T C$ & $(1787,1787)$ & $(1787,1787)$ & $(1787,1787)$ & $(1787,1787)$ & $(1787,1787)$ \\
\hline & \multirow{2}{*}{7} & $N$ & $(3,3)$ & $(3,3)$ & $(3,3)$ & $(3,3)$ & $(3,3)$ \\
\hline & & $T C$ & $(2744,2744)$ & $(2744,2744)$ & $(2744,2744)$ & $(2744,2744)$ & $(2744,2744)$ \\
\hline & \multirow{2}{*}{9} & $N$ & $(3,3)$ & $(3,3)$ & $(3,3)$ & $(3,3)$ & $(3,3)$ \\
\hline & & $T C$ & $(3999,4000)$ & $(3999,4000)$ & $(3999,4000)$ & $(3999,4000)$ & $(3999,4000)$ \\
\hline & \multirow{2}{*}{11} & $N$ & $(3,4)$ & $(4,4)$ & $(5,4)$ & $(5,4)$ & $(5,4)$ \\
\hline & & $T C$ & $(4602,5357)$ & $(4462,5036)$ & $(3831,4740)$ & $(3675,4561)$ & $(3759,4499)$ \\
\hline \multirow{10}{*}{$T$} & \multirow{2}{*}{2} & $N$ & $(3,3)$ & $(4,3)$ & $(5,3)$ & $(7,3)$ & $(6,3)$ \\
\hline & & $T C$ & $(2499,2708)$ & $(2506,2451)$ & $(2142,2235)$ & $(1898,2136)$ & $(2383,2154)$ \\
\hline & \multirow{2}{*}{3} & $N$ & $(4,4)$ & $(4,4)$ & $(5,4)$ & $(5,4)$ & $(5,4)$ \\
\hline & & $T C$ & $(2499,3029)$ & $(2506,2907)$ & $(2142,2859)$ & $(2031,2859)$ & $(1945,2859)$ \\
\hline & \multirow{2}{*}{4} & $N$ & $(3,3)$ & $(3,3)$ & $(3,3)$ & $(3,3)$ & $(3,3)$ \\
\hline & & $T C$ & $(2744,2744)$ & $(2744,2744)$ & $(2744,2744)$ & $(2744,2744)$ & $(2744,2744)$ \\
\hline & \multirow{2}{*}{5} & $N$ & $(3,3)$ & $(3,3)$ & $(3,3)$ & $(3,3)$ & $(3,3)$ \\
\hline & & $T C$ & $(2744,2744)$ & $(2744,2744)$ & $(2744,2744)$ & $(2744,2744)$ & $(2744,2744)$ \\
\hline & \multirow{2}{*}{6} & $N$ & $(3,3)$ & $(3,3)$ & $(3,3)$ & $(3,3)$ & $(3,3)$ \\
\hline & & $T C$ & $(2744,2744)$ & $(2744,2744)$ & $(2744,2744)$ & $(2744,2744)$ & $(2744,2744)$ \\
\hline
\end{tabular}

difference between Algorithms $A_{1}$ and $A_{2}$ for this numerical example.

In order to obtain a fair comparison between these two heuristic algorithms, we change the values of some parameters among $H, T$, and $T_{0}$ to this numerical example and keep the remaining parameters unchanged. Let $(\cdot, \cdot)$ be the calculation results obtained by heuristic Algorithms $A_{1}$ and $A_{2}$, and the corresponding results are shown in Table 1.

From Table 1, we can conclude that

(1) the total cost obtained by each algorithm is more sensitive to the changes of $H$ and $T$ than that of $T_{0}$. Moreover, when $H \leq T$, the solutions obtained by both heuristic algorithms are the optimal solution of model $P$;

(2) when the optimal solution over the finite planning horizon satisfies the restriction on production run time, the solutions obtained by two heuristic algorithms are the same, such as cases $H=5$ and $H=7$;

(3) when $H=11$ or $T=2$ and 3, the total cost obtained by heuristic Algorithm $A_{2}$ first decreases and then increases as $T_{0}$ increases;

(4) the two heuristic algorithms obtain almost the same solution when the finite planning horizon is taken as the unique interval, such as case $H=9$;

(5) the difference of the total cost obtained by two heuristics is at most 914 (cases $T=3$ and $T_{0}=2$ ).

To sum up, for the most part, the total cost obtained by heuristic Algorithm $A_{1}$ is not more than the one obtained by heuristic Algorithm $A_{2}$. It means that heuristic Algorithm $A_{1}$ is superior to heuristic Algorithm $A_{2}$. Hence, we can employ heuristic Algorithm $A_{1}$ to solve our problem.

\section{Conclusions}

An integrated EPQ and preventive maintenance problem under restrictions on production run time and maintenance time is proposed. Based on the assumption that the production run time can not exceed a maximum value and the preventive maintenance time can not less than a minimum value, a mixed integer quadratic programming model is built. The optimal properties of the relaxation model are investigated, and two heuristic algorithms are designed using different techniques. The numerical experiments show that, for the most part, heuristic Algorithm $A_{1}$ is more effective than heuristic Algorithm $A_{2}$.

This paper might be extended in several ways. For example, in the model presented, we assume that the demand rate is constant. However, the models with random demand and dynamic demand are also interesting issues for further research. When there is more than one production facility in the production system, how to determine the production and maintenance decision might be another interesting issue. Finally, it might also be interesting to investigate a supply chain system and reveal the interactions of behaviors among the supply chain members.

\section{Appendix}

Proof of Theorem 5. Let $T(N, 0, H)=\sum_{i=1}^{N}\left(b B_{i}+h I_{i}\right)$ be the total cost of $N$ inventory cycles in interval $[0, H]$. The total 
cost of relaxation model (7)-(9) satisfies $\mathrm{TC}^{\prime}(N, \mathbf{v}, \mathbf{r})=N S+$ $c d H+T(N, 0, H)$. Let $T^{*}(N, 0, H)$ be the minimal cost of $N$ inventory cycles in interval $[0, H]$. According to the Dynamic Programming Principle, we have

$$
\begin{aligned}
T^{*} & (N, 0, H) \\
& =\min _{y \in[0, H]}\left\{T^{*}(N-1,0, y)+T(1, y, H)\right\} .
\end{aligned}
$$

Let $y=H$; we have $T^{*}(N, 0, H) \leq T^{*}(N-1,0, H)$. That means that $T^{*}(N, 0, H)$ is decreasing in $N$.

Next, we prove that $T^{*}(N, 0, H)$ is convex in $N$. Let $H_{1}$ and $H_{2}$ be the points that satisfy $y_{N}^{*}\left(N+1,0, H_{1}\right)=y_{N+1}^{*}(N+$ $\left.2,0, H_{2}\right)=H$. According to the Dynamic Programming Principle, we have

$$
\begin{aligned}
T^{*} & \left(N+1,0, H_{1}\right) \\
& =\min _{y \in\left[0, H_{1}\right]}\left\{T^{*}(N, 0, y)+T\left(1, y, H_{1}\right)\right\} \\
& =T^{*}(N, 0, H)+T\left(1, H, H_{1}\right), \\
T^{*} & \left(N+2,0, H_{2}\right) \\
& =\min _{y \in\left[0, H_{2}\right]}\left\{T^{*}(N+1,0, y)+T\left(1, y, H_{2}\right)\right\} \\
& =T^{*}(N+1,0, H)+T\left(1, H, H_{2}\right) .
\end{aligned}
$$

That means that the minimal value of both $T^{*}\left(N+1,0, H_{1}\right)$ and $T^{*}\left(N+2,0, H_{2}\right)$ is obtained at $y=H$. That is,

$$
\begin{array}{r}
\left.\frac{\partial\left[T^{*}(N, 0, y)+T\left(1, y, H_{1}\right)\right]}{\partial y}\right|_{y=H}=0, \\
\left.\frac{\partial\left[T^{*}(N+1,0, y)+T\left(1, y, H_{2}\right)\right]}{\partial y}\right|_{y=H}=0 .
\end{array}
$$

Since $\partial T(1, y, z) / \partial y=-b d(v-y)$, we have

$$
\begin{aligned}
& \left.\frac{\partial T^{*}(N, 0, y)}{\partial y}\right|_{y=H}=-\left.\frac{\partial T\left(1, y, H_{1}\right)}{\partial y}\right|_{y=H} \\
& =b d\left[v_{N+1}^{*}\left(N+1,0, H_{1}\right)-H\right], \\
& \left.\frac{\partial T^{*}(N+1,0, y)}{\partial y}\right|_{y=H}=-\left.\frac{\partial T\left(1, y, H_{2}\right)}{\partial y}\right|_{y=H} \\
& =b d\left[v_{N+2}^{*}\left(N+2,0, H_{2}\right)-H\right] .
\end{aligned}
$$

Using formula (12), bring $v_{i+1}-r_{i}=(1 / b p)\left[h(p-d)\left(r_{i}-v_{i}\right)+\right.$ $\left.b d\left(v_{i}-r_{i-1}\right)\right]$ to (A.4), and we have

$$
\begin{aligned}
& \left.\frac{\partial T^{*}(N, 0, y)}{\partial y}\right|_{y=H} \\
& =\frac{d}{p}\left[h(p-d)\left(H-v_{N}^{*}\left(N+1,0, H_{1}\right)\right)\right. \\
& \left.+b d\left(v_{N}^{*}\left(N+1,0, H_{1}\right)-r_{N-1}^{*}\left(N+1,0, H_{1}\right)\right)\right], \\
& \left.\frac{\partial T^{*}(N+1,0, y)}{\partial y}\right|_{y=H} \\
& =\frac{d}{p}\left[h(p-d)\left(H-v_{N+1}^{*}\left(N+2,0, H_{2}\right)\right)\right. \\
& \left.+b d\left(v_{N+1}^{*}\left(N+2,0, H_{2}\right)-r_{N}^{*}\left(N+2,0, H_{2}\right)\right)\right] .
\end{aligned}
$$

According to the Dynamic Programming Principle, we have

$$
\begin{aligned}
& v_{N}^{*}\left(N+1,0, H_{1}\right)=v_{N}^{*}\left(N, 0, y_{N}^{*}\left(N+1,0, H_{1}\right)\right) \\
& \quad=v_{N}^{*}(N, 0, H)=v_{N}^{*}, \\
& r_{N-1}^{*}\left(N+1,0, H_{1}\right)=r_{N-1}^{*}\left(N, 0, y_{N}^{*}\left(N+1,0, H_{1}\right)\right) \\
& \quad=r_{N-1}^{*}(N, 0, H)=r_{N-1}^{*}, \\
& v_{N+1}^{*}\left(N+2,0, H_{2}\right) \\
& \quad=v_{N+1}^{*}\left(N+1,0, y_{N+1}^{*}\left(N+2,0, H_{2}\right)\right) \\
& \quad=v_{N+1}^{*}(N+1,0, H)=v_{N+1}^{*}, \\
& r_{N}^{*}\left(N+2,0, H_{2}\right) \\
& \quad=r_{N}^{*}\left(N+1,0, y_{N+1}^{*}\left(N+2,0, H_{2}\right)\right) \\
& \quad=r_{N}^{*}(N+1,0, H)=r_{N}^{*} .
\end{aligned}
$$

Since $v_{N}^{*}(N, 0, H) \leq v_{N+1}^{*}(N+1,0, H)$ and $r_{N-1}^{*}(N, 0, H) \leq$ $r_{N}^{*}(N+1,0, H)$, we have

$$
\begin{aligned}
& \left.\frac{\partial T^{*}(N+1,0, y)}{\partial y}\right|_{y=H}-\left.\frac{\partial T^{*}(N, 0, y)}{\partial y}\right|_{y=H} \\
& =-\frac{p}{d}\left[(h(p-d)-b d)\left(v_{N+1}^{*}-v_{N}^{*}\right)\right. \\
& \left.+b d\left(r_{N}^{*}-r_{N-1}^{*}\right)\right] \leq 0 .
\end{aligned}
$$

The above inequality holds, since $h(p-d)-b d \geq 0$. Hence, $T^{*}(N+1,0, H)-T^{*}(N, 0, H)$ is decreasing in $H$; that is, $T^{*}\left(N+1,0, H_{1}\right)-T^{*}\left(N, 0, H_{1}\right) \leq T^{*}(N+1,0, H)-$ $T^{*}(N, 0, H)$.

Now, we prove that $T^{*}(N, 0, H)-T^{*}(N-1,0, H) \leq$ $T^{*}\left(N+1,0, H_{1}\right)-T^{*}\left(N, 0, H_{1}\right)$. 


$$
\begin{aligned}
T^{*}( & \left.+1,0, H_{1}\right)-T^{*}\left(N, 0, H_{1}\right) \\
= & \min _{y \in\left[0, H_{1}\right]}\left\{T^{*}(N, 0, y)+T\left(1, y, H_{1}\right)\right\} \\
& \quad-\min _{y \in\left[0, H_{1}\right]}\left\{T^{*}(N-1,0, y)+T\left(1, y, H_{1}\right)\right\} \\
= & T^{*}(N, 0, H)+T\left(1, H, H_{1}\right) \\
& \quad-\min _{y \in\left[0, H_{1}\right]}\left\{T^{*}(N-1,0, y)+T\left(1, y, H_{1}\right)\right\} \\
\geq & T^{*}(N, 0, H)+T\left(1, H, H_{1}\right) \\
& -\left[T^{*}(N-1,0, H)+T\left(1, H, H_{1}\right)\right] \\
= & T^{*}(N, 0, H)-T^{*}(N-1,0, H) .
\end{aligned}
$$

Hence,

$$
\begin{aligned}
T^{*} & (N, 0, H)-T^{*}(N-1,0, H) \\
& \leq T^{*}\left(N+1,0, H_{1}\right)-T^{*}\left(N, 0, H_{1}\right) \\
& \leq T^{*}(N+1,0, H)-T^{*}(N, 0, H) .
\end{aligned}
$$

That means that $T^{*}(N, 0, H)$ is a convex function of $N$. Since $N S+c d H$ is linear increasing in $N$, and $\operatorname{TC}^{\prime}(N, \mathbf{v}, \mathbf{r})$ is also a convex function on $N$.

\section{Notations}

\section{Parameters}

$H$ : The length of the finite planning horizon

$d$ : Demand rate

$p:$ Production rate, $p>d$

$T$ : The maximum production run time for each production phase

$T_{0}$ : The minimum maintenance time for each preventive maintenance

$S_{0}$ : The fixed maintenance cost and setup cost that occurred when production facility is turned on

S: Fixed cost that occurred when a new batch is organized to be produced

$c$ : Unit production cost

$h$ : Unit holding cost

$b$ : Unit shortage penalty cost, $b \geq h$. (Furthermore, we assume that the holding cost of the surplus products per unit time is less than the shortage penalty cost per unit time; that is, $h(p-d) \geq b d$.)

\section{Variables}

$N_{0}$ : The total number of production phases

$N$ : The total number of production cycles

$u_{i}$ : The start time of $i$ th production cycle, $i=1, \ldots, N, u_{1}=0$

$v_{i}$ : The end time of $i$ th production cycle, $i=1, \ldots, N$

$r_{i}$ : The time when the inventory level drops to zero for $i$ th time, $i=1, \ldots, N, r_{0}=0$.

\section{Competing Interests}

The authors declare that they have no competing interests.

\section{Acknowledgments}

This work was supported by the National Natural Science Foundation of China (Grant no. 71371107) and the Humanities and Social Sciences Research Youth Foundation of Ministry of Education of China (Grant no. 14YJCZH171).

\section{References}

[1] M. K. Salameh and M. Y. Jaber, "Economic production quantity model for items with imperfect quality," International Journal of Production Economics, vol. 64, no. 1-3, pp. 59-64, 2000.

[2] K.-J. Chung and Y.-F. Huang, "The optimal cycle time for EPQ inventory model under permissible delay in payments," International Journal of Production Economics, vol. 84, no. 3, pp. 307-318, 2003.

[3] L. E. Cárdenas-Barrón, "Economic production quantity with rework process at a single-stage manufacturing system with planned backorders," Computers \& Industrial Engineering, vol. 57, no. 3, pp. 1105-1113, 2009.

[4] A. Jeang, "Economic production order quantity and quality," International Journal of Production Research, vol. 49, no. 6, pp. 1753-1783, 2011.

[5] S.-D. Lee and C.-M. Yang, "An economic production quantity model with a positive resetup point under random demand," Applied Mathematical Modelling, vol. 37, no. 5, pp. 3340-3354, 2013.

[6] J. Mezei and K.-M. Björk, "An economic production quantity problem with backorders and fuzzy cycle times," Journal of Intelligent and Fuzzy Systems, vol. 28, no. 4, pp. 1861-1868, 2015.

[7] S.-D. Lee, C.-M. Yang, and S.-C. Lan, "Economic lot sizing in a production system with random demand," International Journal of Systems Science. Principles and Applications of Systems and Integration, vol. 47, no. 5, pp. 1142-1154, 2016.

[8] H. Groenevelt, L. Pintelon, and A. Seidmann, "Production lot sizing with machine breakdowns," Management Science, vol. 38, no. 1, pp. 104-123, 1992.

[9] N. E. Abboud, "A simple approximation of the EMQ model with Poisson machine failures," Production Planning and Control, vol. 8, no. 4, pp. 385-397, 1997.

[10] G. C. Lin and D.-C. Gong, "On a production-inventory system of deteriorating items subject to random machine breakdowns with a fixed repair time," Mathematical and Computer Modelling, vol. 43, no. 7-8, pp. 920-932, 2006.

[11] S. W. Chiu, S.-L. Wang, and Y.-S. P. Chiu, "Determining the optimal run time for EPQ model with scrap, rework, and stochastic breakdowns," European Journal of Operational Research, vol. 180, no. 2, pp. 664-676, 2007.

[12] Y.-S. P. Chiu, C.-Y. Tseng, W.-C. Liu, and C.-K. Ting, "Economic manufacturing quantity model with imperfect rework and random breakdown under abort/resume policy," Proceedings of the Institution of Mechanical Engineers, Part B: Journal of Engineering Manufacture, vol. 223, no. 2, pp. 183-194, 2009.

[13] O. Prakash, A. R. Roy, and A. Goswami, "Stochastic manufacturing system with process deterioration and machine breakdown," International Journal of Systems Science, vol. 45, no. 12, pp. 2539-2551, 2014. 
[14] P.-S. Ting and K.-J. Chung, "On the convexity for the expected total cost per unit time of the EPQ model with scrap, rework and stochastic machine breakdown," Applied Mathematical Modelling, vol. 38, no. 7-8, pp. 2296-2301, 2014.

[15] R. D. Meller and D. S. Kim, "The impact of preventive maintenance on system cost and buffer size," European Journal of Operational Research, vol. 95, no. 3, pp. 577-591, 1996.

[16] B. C. Giri and T. Dohi, "Computational aspects of an extended EMQ model with variable production rate," Computers and Operations Research, vol. 32, no. 12, pp. 3143-3161, 2005.

[17] G.-L. Liao, Y. H. Chen, and S.-H. Sheu, "Optimal economic production quantity policy for imperfect process with imperfect repair and maintenance," European Journal of Operational Research, vol. 195, no. 2, pp. 348-357, 2009.

[18] G.-L. Liao and S.-H. Sheu, "Economic production quantity model for randomly failing production process with minimal repair and imperfect maintenance," International Journal of Production Economics, vol. 130, no. 1, pp. 118-124, 2011.

[19] T.-H. Lee, "Optimal production run length and maintenance schedule for a deteriorating production system," International Journal of Advanced Manufacturing Technology, vol. 43, no. 910, pp. 959-963, 2009.

[20] Y.-H. Lin, J.-M. Chen, and Y.-C. Chen, "The impact of inspection errors, imperfect maintenance and minimal repairs on an imperfect production system," Mathematical and Computer Modelling, vol. 53, no. 9-10, pp. 1680-1691, 2011.

[21] S. S. Sana, "Preventive maintenance and optimal buffer inventory for products sold with warranty in an imperfect production system," International Journal of Production Research, vol. 50, no. 23, pp. 6763-6774, 2012.

[22] G. A. Widyadana and H. M. Wee, "An economic production quantity model for deteriorating items with preventive maintenance policy and random machine breakdown," International Journal of Systems Science, vol. 43, no. 10, pp. 1870-1882, 2012.

[23] H. M. Wee and G. A. Widyadana, "Economic production quantity models for deteriorating items with rework and stochastic preventive maintenance time," International Journal of Production Research, vol. 50, no. 11, pp. 2940-2952, 2012.

[24] H. M. Wee and G. A. Widyadana, "A production model for deteriorating items with stochastic preventive maintenance time and rework process with FIFO rule," Omega, vol. 41, no. 6, pp. 941-954, 2013.

[25] G.-L. Liao, "Joint production and maintenance strategy for economic production quantity model with imperfect production processes," Journal of Intelligent Manufacturing, vol. 24, no. 6, pp. 1229-1240, 2013.

[26] Y.-C. Tsao, "A piecewise nonlinear optimization for a production-inventory model under maintenance, variable setup costs, and trade credits," Annals of Operations Research, vol. 233, pp. 465-481, 2015.

[27] S. Y. Gan, Z. S. Zhang, Y. F. Zhou, and J. F. Shi, "Joint optimization of maintenance, buffer, and spare parts for a production system," Applied Mathematical Modelling, vol. 39, no. 19, pp. 6032-6042, 2015.

[28] B. Emami-Mehrgani, W. P. Neumann, S. Nadeau, and M. Bazrafshan, "Considering human error in optimizing production and corrective and preventive maintenance policies for manufacturing systems," Applied Mathematical Modelling. Simulation and Computation for Engineering and Environmental Systems, vol. 40, no. 3, pp. 2056-2074, 2016.

[29] M. Nourelfath, N. Nahas, and M. Ben-Daya, "Integrated preventive maintenance and production decisions for imperfect processes," Reliability Engineering \& System Safety, vol. 148, pp. 21-31, 2016.

[30] R. E. Bellman, Dynamic Programming, Princeton University Press, Princeton, NJ, USA, 1957. 


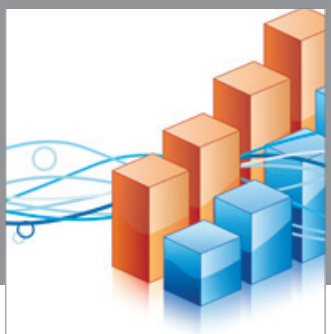

Advances in

Operations Research

vatem alat4

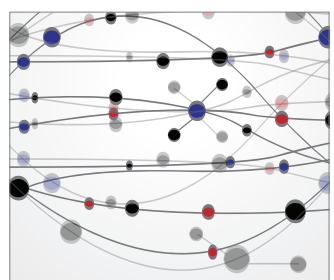

\section{The Scientific} World Journal
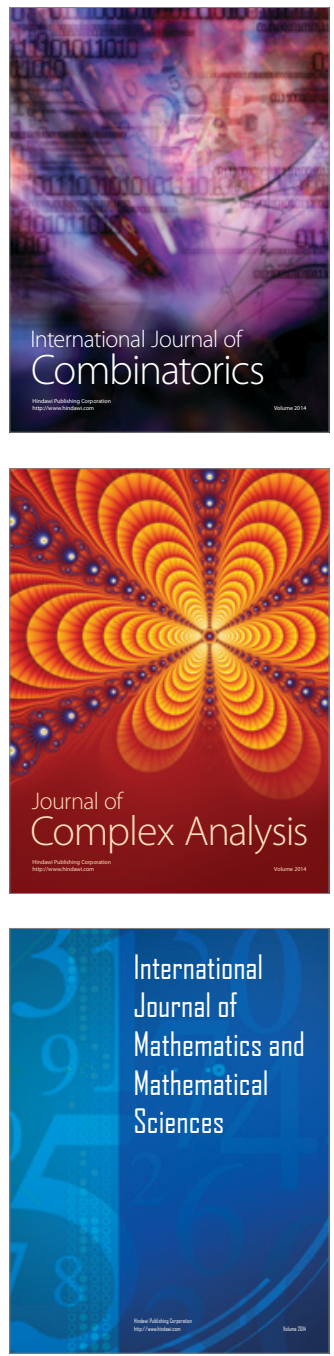
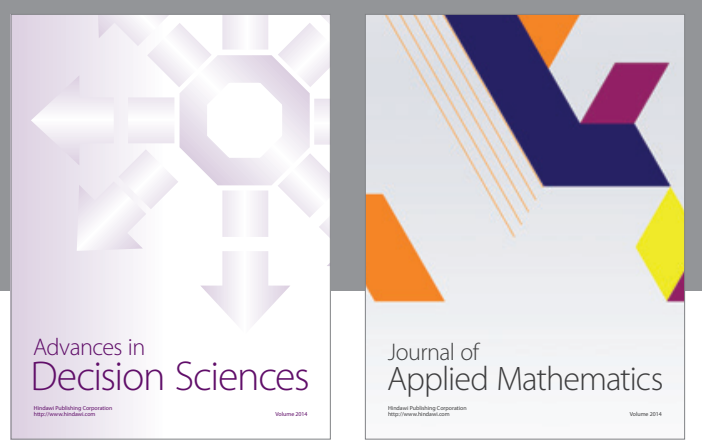

Algebra

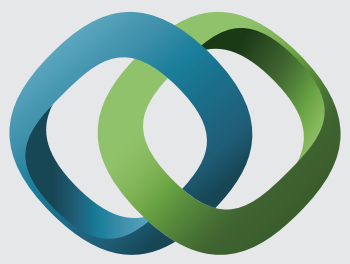

\section{Hindawi}

Submit your manuscripts at

http://www.hindawi.com
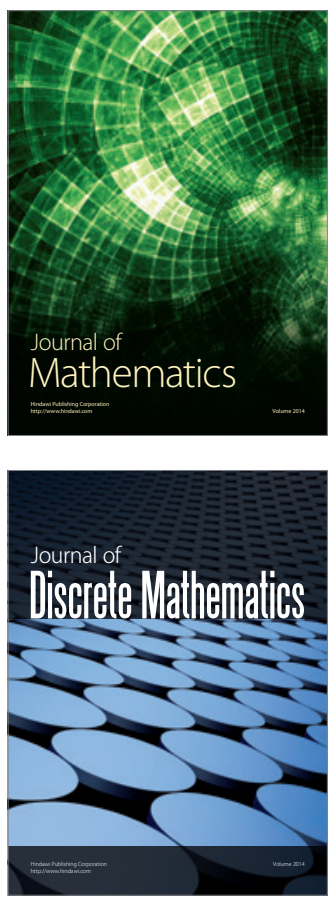

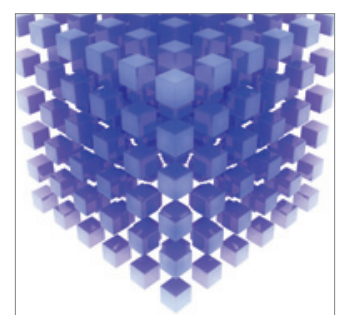

Mathematical Problems in Engineering
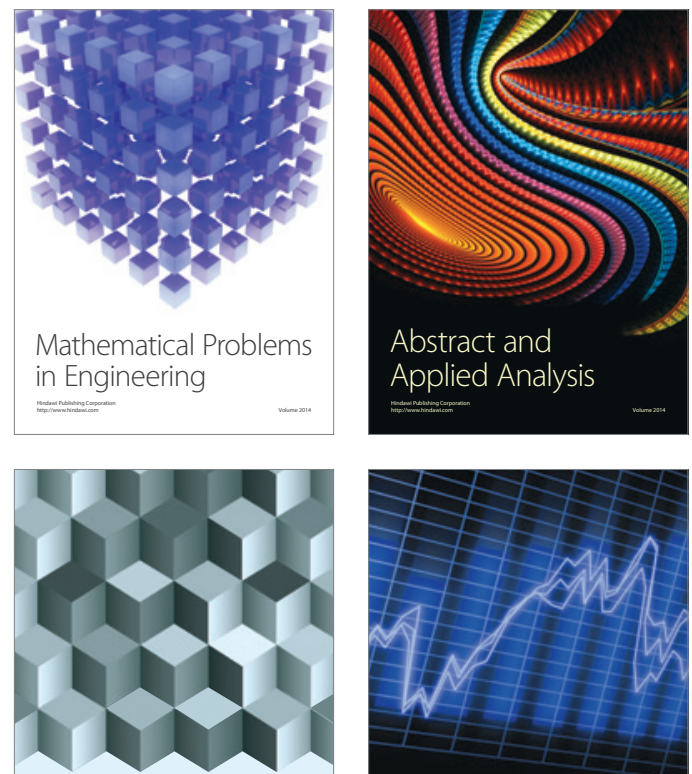

Journal of

Function Spaces

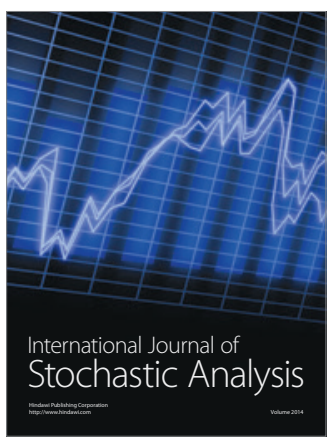

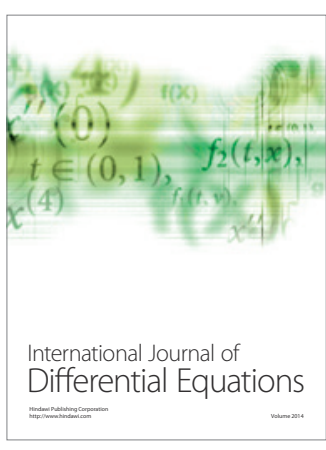
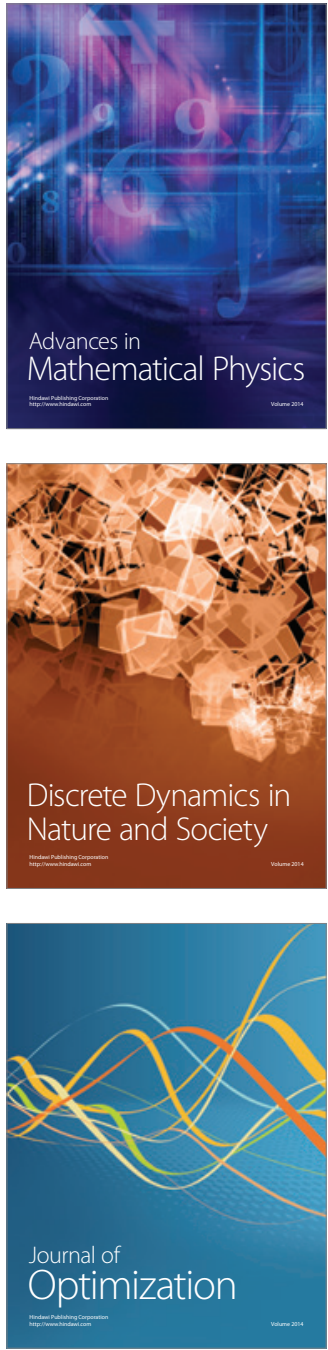